$\begin{array}{ll} & \text { Preprints are preliminary reports that have not undergone peer review. } \\ \text { Research Square } & \text { They should not be considered conclusive, used to inform clinical practice, } \\ \text { or referenced by the media as validated information. }\end{array}$

\title{
Intimate Partner Violence and Its Association With HIV Testing Among Filipino Women: Results From the 2017 Philippine National Demographic and Health Survey
}

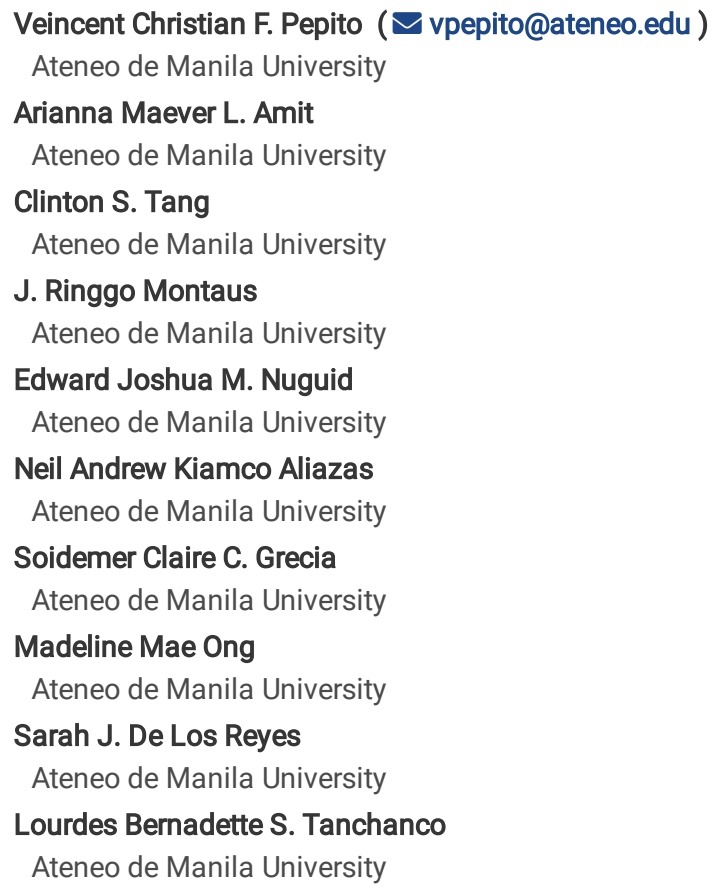




\section{Abstract}

Background: The Philippines is one of the few countries in the world where incidence of both HIV and intimate partner violence (IPV) continue to rise. There is conflicting evidence on the association between intimate partner violence (IPV) with HIV testing however, and such an analysis involving Filipino respondents has not been carried out before. Our paper thus aims to study the relationship between IPV and HIV testing. As a secondary objective, we aim to assess the effect of confiding their IPV experiences to other people on HIV testing.

Methods: We used data from the 2017 Philippine National Demographic and Health Survey, only including participants on its domestic violence module. We used logistic regression methods for survey data to study the associations of interest.

Results: Out of 17,968 respondents, around $16 \%$ of respondents reported experiencing any form of IPV, $13 \%$ reported experiencing emotional violence, $7 \%$ reported experiencing physical violence, and $3 \%$ reported experiencing sexual violence, with even a smaller percentage confiding their experience to other people. After adjusting for confounders, those who have experienced emotional violence have a higher odds of HIV testing as compared to those who have never experienced emotional violence. The odds of HIV testing are lower among those who have experienced any form of violence, physical violence, and sexual violence as compared to respondents who have not experienced any form of violence or these specific types of violence. More worryingly, the odds of HIV testing among victims of these types of IPV and have confided with other people are lower than those who have never been a victim of IPV or those who have not confided being a victim of IPV. None of the associations of interest have a statistically significant result.

Conclusions: Our results suggest that there is a need to further improve access to HIV testing services among victims of IPV by making tests for HIV and other STIs part of the standard of care for IPV victims. By doing so, we can meet the 'first 90' of the 90-90-90 UNAIDS targets.

\section{Introduction}

Intimate partner violence (IPV), defined as an act of physical, sexual, or psychological violence caused by a current or former partner that does not require sexual intimacy (1), and high incidence of human immunodeficiency virus (HIV) infections are two of the most pressing health issues encountered by Filipino women today $(2,3)$. The Philippines remains one of the few in the world where the incidence of HIV is rapidly increasing $(2)$. On average, there are more than 30 new cases of HIV that are diagnosed in the country every day; around two of these are women. This rapid increase in HIV incidence can be attributed to a wide host of socio-behavioral and personal factors, one of which is personal aversity to HIV testing (4-6). Such aversity to HIV testing means that current HIV statistics underestimate the actual case burden in the country by as much as one-third (6). Despite recent developments to promote its uptake $(7,8)$, low uptake of HIV testing remains to be a problem in the Philippines as only $5 \%$ of Filipino women have ever tested for HIV (9). This falls short of the $90 \%$ testing target of the Philippine Department of Health, which corresponds to the first ' 90 ' of the $90-90-90$ target of the Joint United Nations Program on HIV/AIDS (UNAIDS) (6). In addition to aversion to HIV testing, IPV has also been previously linked to increased risk of contracting HIV among women (10), including Filipino women (11). According to the 2017 Philippine National Demographic and Health Survey (NDHS), $14.7 \%$ of ever-married Filipino women have experienced any form of IPV in the past 12 months (9).

One study, focusing solely on the determinants of IPV in the country found that the prevalence of physical violence in Cebu, Philippines is $13.0 \%$. The same study also concluded that household wealth, urban residence, husband's religion, household income distribution, household decision making, women's empowerment, and use of modern contraceptives have been associated with IPV (12). In other countries, previous studies have identified that depressive symptoms, history of childhood abuse, unplanned and unwanted pregnancies, multiple sex partners, alcohol consumption and substance abuse, controlling behavior of husband, being HIV positive and perceptions of stigma due to HIV have been found to be associated with the IPV (1320).

Another study in the Philippines focused on the determinants of HIV testing among Filipino women in a nationally representative survey and found that having tertiary education, living with partner, tobacco use, socio-economic status, access to television and internet, living in rural areas, and being a Muslim is associated with ever testing for HIV (21). A systematic review has also identified a host of healthcare provider factors, such as perceived risk of infection and fear and worries, and institutional factors, such as accessibility of health services and scarcity of human and health resources that work to encourage or discourage HIV testing (22).

In previous studies exploring the association between IPV and HIV testing, evidence is conflicting. One study in the United States shows that experiencing physical and sexual IPV is positively associated with HIV testing (23), but another study states that IPV is negatively associated with selftesting for HIV in Kenya (24). Another study in the United States did not find any evidence of association between IPV and HIV testing (25). The conflicting evidence from other countries, together with the lack of studies examining the association between IPV and HIV testing in the Philippines, highlights a gap which should be addressed to promote HIV testing among Filipino women. Furthermore, none of the studies cited yet have assessed whether a victim of IPV who has confided her IPV experiences will be more (or less) likely to test. This gap represents a potential point to explore where response programs on victims of domestic violence could be expanded to promote HIV testing services as well.

Our paper aims to address these literature gaps by studying the association between experiencing any type of IPV, experiencing specific types of IPV (physical, sexual, and emotional violence) and having HIV testing among Filipino women. The results of our analysis can be used to tailor interventions to promote HIV testing among Filipino women, specifically among those who have experienced IPV. 


\section{Methods}

\section{Study population and sampling method}

The 2017 Philippine NDHS is a nationally representative survey whose primary objective is to provide up-to-date estimates of basic demographic and health indicators (9). The survey utilized a two-stage stratified design utilizing the Master Sampling Frame designed by the Philippine Statistics Authority. The strata used were 117 major sampling domains in the country (81 provinces, 33 highly urbanized cities and 3 special areas). The first stage sampling involved the systematic selection of 1,250 primary sampling units throughout the entire Philippines. Such primary sampling units can be barangays (Philippine equivalent of village), a portion of a large barangay or two or more adjacent small barangays. Once these primary sampling units were selected, either 20 or 26 housing units were systematically random sampled. Non-replacement sampling was utilized to prevent bias. Survey weights were then computed. All women aged 15-49 years old who were either permanent residents of the selected households or visitors who stayed in the households the night before the survey were eligible to be interviewed. Among these eligible women for each household, one was randomly selected for a module on domestic violence. Separate survey weights were computed for those who participated in the domestic violence module. Other details of the sampling method utilized by the survey is available in its published manuscript (9). For this analysis, only those that participated in the domestic violence module are included.

\section{Data collection, definition of variables, and data management}

Two questionnaires administered using tablet computers by trained field interviewers were used for the 2017 NDHS. The questionnaires were translated to six local languages commonly spoken throughout the Philippines: Tagalog, Cebuano, Ilokano, Bikol, Hiligaynon, and Waray and were then pre-tested on April 21, 2017. Actual data collection was done on August 14 to October 27, 2017. Data processing began shortly after initiation of data collection, and a clean dataset was prepared by late 2017 (9). For this analysis, we will use the Individual Recode dataset of the 2017 Philippine NDHS.

The survey collected data on a wide range of socio-demographic variables, fertility levels and preferences, awareness and use of family planning methods, breastfeeding, maternal and child health, child mortality, awareness and behavior regarding HIV/AIDS, women's empowerment, domestic violence, and other health-related issues (9). However, for this analysis, we only focus on HIV/AIDS, women's empowerment, domestic violence, and socio-demographic variables. The exposure variables for this analysis are experiencing any form of emotional violence, physical violence, and sexual violence, which were aggregated to form another variable on experiencing any form of intimate partner violence. These exposure variables have three categories: the baseline category would be not experiencing any types of violence. The second category would be experiencing the said types of violence but not telling anybody about it, and the third category would be experiencing the said types of violence and confiding it with somebody. This way, we could model the effect of experiencing the different forms of domestic violence and the effect of confiding their experiences with somebody on our outcome variable. The outcome variable in this analysis is having ever tested for HIV, dichotomized into never having tested for HIV and having ever tested for HIV. The probable confounders that were assessed in this analysis were hurting partner, alcohol intake of partner, respondent's father beats mother, educational attainment of respondent, educational attainment of partner, domicile, religion, access to newspaper, radio, television, and internet, wealth index, employment status, marital status, condom use, tobacco use, alcohol use, age of respondent, household size, age at first sex of respondent, age of partner, number of lifetime sexual partners, number of children, HIV knowledge score, tolerance to domestic violence score, women's empowerment score, and extent of controlling behavior of spouse (13-21).

HIV knowledge score, tolerance to domestic violence score, women's empowerment score, and extent of controlling behavior of spouse are score variables that were aggregated from many items across the NDHS dataset. HIV knowledge score is aggregated from the answers of respondents on eight yes or no items from the NDHS: (a) reduce risk of getting HIV by having sex with only one partner who has no other partners; (b) reducing risk of getting HIV by always using condom; (c) can get HIV from mosquito bites; (d) can get HIV by sharing food with somebody; (e) a healthy looking person can have HIV; (f) HIV can be transmitted during pregnancy; (g) HIV can be transmitted during delivery; (h) HIV transmitted by breastfeeding. For each item a respondent gets correctly, the respondent gets one point. The points from each item were added to comprise the HIV knowledge score, thus, those who have a higher HIV knowledge score have greater HIV knowledge as compared to those who have low knowledge. Tolerance to domestic violence score is aggregated from five items where a respondent is asked if beating is justified if wife: (a) goes out without telling husband; (b) neglects the children; (c) argues with husband; (d) refuses to have sex with husband; and (e) burns food. For each item that a respondent has said 'yes', she will get one point. The points from each item are added to comprise the tolerance to domestic violence score. Therefore, respondents with a higher domestic violence score find more reasons to tolerate domestic violence as compared to respondents with a lower domestic violence score. Women's empowerment scores are aggregated from four items: (a) who decides on large household purchases; (b) who decides on visits to family or relatives; (c) who usually decides on what to do with money that husband earns; and (d) who usually decides on respondent's health care. For each of these items, a respondent gets two points if she answered, "respondent alone", one point if she answered that both she and her husband make the decision together, and no points if she is not involved in any decision-making. The points from these items were added to comprise the women's empowerment score, with a higher score indicating that the respondent has more freedom in making decisions for herself. Lastly, there is an aggregate variable to measure the extent of the partner's controlling behavior. The variable is aggregated from the following five items: (a) husband/partner jealous if respondent talks with other men; (b) husband/partner accuses respondent of unfaithfulness; (c) husband/partner does not permit respondent to meet female friends; (d) husband/partner tries to limit respondent's contact with family; and (e) husband/partner insists on knowing where respondent is. For each yes to these items, a respondent gets one point, and the higher the score for this part, the more controlling her husband is. Lastly, we also recoded variables, such as civil status, religion, and condom use to ensure that each category would have sufficient respondents. 


\section{Data Analysis}

All data management and analyses were carried out in Stata 14.0 IC (26) and we used a level of significance at 0.05 (27). After data cleaning and recoding, we declared our dataset as survey data using the separate weighting scheme for those who participated in the domestic violence module as sampling weight. This way, we restrict the analyses to those who have participated in the said domestic violence module. All our subsequent analyses are weighted, except for analyses that do not have an option for weighting. Thus, proportions, means odds ratios, and p-values, except for tests of normality and rank-sum tests, were weighted. However, we still showed the number of observations, which are unweighted. Once we declared our dataset as survey data, we ran descriptive statistics for all variables of interest. We identified the proportions and frequencies of the categories for each of our categorical variables. For our continuous variables, we described their range, distribution, and the appropriate measure of central tendency. We also described the number of respondents with missing data for each variable under study.

After describing our data, we cross-tabulated the exposure variables and probable confounders with the outcome variable and note the p-values of the Pearson's chi-square test and crude odds ratios. For our probable confounders which are continuous variables, we performed either Wald test for normally distributed variables or rank-sum test for skewed variables, to assess their association with the outcome. We repeated these cross-tabulations to assess the association of the outcomes and confounders with each of the exposure variables and identify confounders to control for in our analyses later. Before multivariate analysis, we ran a correlation matrix to assess potential collinearity between the variables. We also excluded respondents from the analyses with those who have missing data in any of the variables of interest by using Stata's subpop function to ensure that estimates and standard errors are still computed correctly. After this, we noted how many observations were excluded from the analyses.

We used logistic regression for survey data in estimating both our crude and adjusted estimates. Since experiencing emotional violence, sexual violence, and physical violence are in the same causal pathway as experiencing any form of interpersonal violence, we ran two regression models: one where the exposure to the three specific forms of IPV were the exposure variables, while the other is where experiencing any form of IPV is the main exposure variable. We first made the regression model for the latter, fitting the exposure variables first and then the confounding variables. Once all known confounders have been fitted, we fit the remaining variables into the model one by one, starting with the variable with the smallest $\mathrm{p}$-value in the cross-tabulations of exposures and probable confounders with the outcome. As variables were entered into the model, we noted the change in the adjusted odds ratio in the association between the main exposure variable and the outcome variable. If one of the variables being assessed caused the adjusted odds ratio to change by greater than $10 \%$ as compared to the full model, we retained the assessed variable into the model, otherwise, we excluded it. Once we have assessed all variables, we used the same set of confounding variables to make the model for the one with the three specific forms of IPV as exposure variables. We report the adjusted odds ratio for each of the two models separately.

\section{Results}

A total of 17,968 women aged 15 to 49 years old from across the Philippines participated in the domestic violence module of the 2017 Philippine NDHS and were included in this analysis. Around $80 \%$ of them are Roman Catholics, while around $60 \%$ are either married or living with their partner, and around $85 \%$ finished high school or college. A little less than half of them were employed in the previous year. Most of them have access to various forms of media at least once a week. Only a quarter drink alcoholic beverage regularly, and only $5 \%$ use tobacco. There is a more even distribution of respondents in terms of urbanicity and socio-economic status. Most of them have good knowledge on HIV, with the population having a mean score of 5.18 (95\% Cl: 5.12-5.23), and a median of 5 with a left-skewed distribution. They also have a low tolerance to domestic violence, with a mean tolerance to domestic violence score of 0.21 (95\% $\mathrm{Cl}: 0.20-0.23$ ), a median of 0 , and a right-skewed distribution. Most of the respondents did not have controlling partners, with an average of $0.72(95 \% \mathrm{Cl}: 0.69-0.75$; median $=0$, right-skewed distribution) aspects of their life that they feel are controlled by their partner. However, their women's empowerment score is low, with an average of 4.81 (95\% Cl: 4.77-4.87), a median of 4 and a right-skewed distribution. Of this population, a total of 3,389 (15.67\%) respondents reported experiencing intimate partner violence of any form, with 2,517 (11.35\%), 1,122 (5.22\%) and 484 (2.12\%) experiencing emotional, physical, and sexual violence, respectively, and have not confided it with anybody. In addition to these, there are $386(1.75 \%), 364$ (1.84\%), and 107 (0.4\%), who have experienced emotional, physical, and sexual violence, respectively, and have confided it with somebody else. Out of 17,968 respondents, only 594 respondents (4.31\%) reported having ever been tested for HIV.

Without controlling for confounders, we found that exposure to any form of IPV, as well as to each of the specific types of IPV, have a lower odds of HIV testing as compared to those who were not victims of IPV. The odds of HIV testing are even lower among victims of any form of IPV, physical violence, and sexual violence who have confided it with other people as compared to those who have not confided it. Among the other categorical variables, there is strong evidence that: having a partner who consumes alcoholic beverages regularly, having a high educational attainment, being Aglipay, Islam or Other Christian, having a partner with high educational attainment, living in an urban area, having weekly access to newspaper or magazine, radio, television and internet, belonging to a higher socio-economic class, working in the previous year, living with partner or being widowed, divorced, or separated, using tobacco or alcohol regularly, are all associated with greater odds of HIV testing (Table 1). Among the other continuous variables there is strong evidence that: being older, having a younger partner, higher HIV knowledge, and having a lower tolerance to domestic violence, have a greater odds of HIV testing (Table 2). 
Table 1

Cross-tabulations of categorical exposures and probable confounders with HIV testing $(n=17,968)$.

\begin{tabular}{|c|c|c|c|c|c|}
\hline & $\begin{array}{l}\text { Never been tested for } \\
\text { HIV }\end{array}$ & $\begin{array}{l}\text { Have been tested for } \\
\text { HIV }\end{array}$ & $\begin{array}{l}\text { p- } \\
\text { value }\end{array}$ & $\begin{array}{l}\text { Crude OR } \\
\text { (with 95\% } \\
\text { Cl) }\end{array}$ & $\begin{array}{l}\text { p-value of } \\
\text { OR }\end{array}$ \\
\hline \multicolumn{6}{|l|}{ Any Violence } \\
\hline \multirow[t]{2}{*}{ Has not experienced said violence } & 9,487 & 339 & \multirow[t]{6}{*}{0.81} & 1 & \\
\hline & $(94.73)$ & $(5.27)$ & & & \\
\hline \multirow[t]{2}{*}{ Experienced violence but did not confide } & 2,787 & 121 & & 0.94 & 0.71 \\
\hline & $(95.02)$ & $(4.98)$ & & $(0.68-1.30)$ & \\
\hline \multirow[t]{2}{*}{ Experienced violence but confided } & 457 & 24 & & 0.83 & 0.57 \\
\hline & $(95.59)$ & $(4.41)$ & & $(0.43-1.58)$ & \\
\hline \multirow[t]{2}{*}{ Missing } & 4,643 & 110 & & & \\
\hline & $(97.27)$ & $(2.73)$ & & & \\
\hline \multicolumn{6}{|l|}{ Emotional Violence } \\
\hline \multirow[t]{2}{*}{ Has not experienced said violence } & 9,955 & 357 & 0.97 & 1 & \\
\hline & $(94.78)$ & $(5.22)$ & & & \\
\hline \multirow[t]{2}{*}{ Experienced violence but did not confide } & 2,409 & 108 & & 0.96 & 0.82 \\
\hline & $(94.98)$ & $(5.02)$ & & $(0.67-1.37)$ & \\
\hline \multirow[t]{2}{*}{ Experienced violence but confided } & 367 & 19 & & 0.97 & 0.93 \\
\hline & $(94.94)$ & $(5.06)$ & & $(0.47-1.98)$ & \\
\hline \multirow[t]{2}{*}{ Missing } & 4,643 & 110 & & & \\
\hline & $(97.27)$ & $(2.73)$ & & & \\
\hline \multicolumn{6}{|l|}{ Physical Violence } \\
\hline \multirow[t]{2}{*}{ Has not experienced said violence } & 11,308 & 421 & 0.75 & 1 & \\
\hline & $(94.76)$ & $(5.24)$ & & & \\
\hline \multirow[t]{2}{*}{ Experienced violence but did not confide } & 1,072 & 50 & & 0.95 & 0.86 \\
\hline & $(94.99)$ & $(5.01)$ & & $(0.57-1.60)$ & \\
\hline \multirow[t]{2}{*}{ Experienced violence but confided } & 351 & 13 & & 0.70 & 0.44 \\
\hline & $(96.26)$ & $(3.74)$ & & $(0.29-1.71)$ & \\
\hline \multirow[t]{2}{*}{ Missing } & 4,643 & 110 & & & \\
\hline & $(97.27)$ & $(2.73)$ & & & \\
\hline \multicolumn{6}{|l|}{ Sexual violence } \\
\hline \multirow[t]{2}{*}{ Has not experienced said violence } & 12,162 & 462 & 0.21 & 1 & \\
\hline & $(94.75)$ & $(5.25)$ & & & \\
\hline \multirow[t]{2}{*}{ Experienced violence but did not confide } & 467 & 17 & & 0.70 & 0.25 \\
\hline & $(96.29)$ & $(3.71)$ & & $(0.37-1.30)$ & \\
\hline \multirow[t]{2}{*}{ Experienced violence but confided } & 102 & 5 & & 0.45 & 0.15 \\
\hline & $(97.55)$ & $(2.45)$ & & $(0.15-1.34)$ & \\
\hline \multirow[t]{2}{*}{ Missing } & 4,643 & 110 & & & \\
\hline & $(97.27)$ & $(2.73)$ & & & \\
\hline
\end{tabular}




\begin{tabular}{|c|c|c|c|c|c|}
\hline & $\begin{array}{l}\text { Never been tested for } \\
\text { HIV }\end{array}$ & $\begin{array}{l}\text { Have been tested for } \\
\text { HIV }\end{array}$ & $\begin{array}{l}\mathrm{p}- \\
\text { value }\end{array}$ & $\begin{array}{l}\text { Crude OR } \\
\text { (with 95\% } \\
\text { Cl) }\end{array}$ & $\begin{array}{l}\text { p-value of } \\
\text { OR }\end{array}$ \\
\hline \multirow[t]{2}{*}{ No } & 11,205 & 422 & 0.89 & 1 & \\
\hline & $(94.80)$ & $(5.20)$ & & & \\
\hline \multirow[t]{2}{*}{ Yes } & 1,526 & 62 & & 0.96 & 0.89 \\
\hline & $(94.99)$ & $(5.01)$ & & $(0.56-1.64)$ & \\
\hline \multirow[t]{2}{*}{ Missing } & 4,643 & 110 & & & \\
\hline & $(97.27)$ & $(2.73)$ & & & \\
\hline \multicolumn{6}{|c|}{ Alcohol intake of partner } \\
\hline \multirow[t]{2}{*}{ No } & 4,981 & 151 & $<0.01$ & 1 & \\
\hline & $(96.38)$ & $(3.62)$ & & & \\
\hline \multirow[t]{2}{*}{ Yes } & 7,750 & 333 & & 1.71 & $<0.01$ \\
\hline & $(93.96)$ & $(6.04)$ & & $(1.24-2.37)$ & \\
\hline \multirow[t]{2}{*}{ Missing } & 4,643 & 110 & & & \\
\hline & $(97.27)$ & $(2.73)$ & & & \\
\hline \multicolumn{6}{|c|}{ Respondent's father beats mother } \\
\hline \multirow[t]{2}{*}{ No } & 13,906 & 461 & 0.86 & 1 & \\
\hline & $(95.69)$ & $(4.31)$ & & & \\
\hline \multirow[t]{2}{*}{ Yes } & 3,091 & 119 & & 0.96 & 0.86 \\
\hline & $(95.83)$ & $(4.17)$ & & $(0.64-1.45)$ & \\
\hline \multirow[t]{2}{*}{ Missing } & 377 & 14 & & & \\
\hline & $(94.59)$ & $(5.41)$ & & & \\
\hline \multicolumn{6}{|c|}{ Educational attainment of respondent } \\
\hline \multirow[t]{2}{*}{ No education } & 231 & 0 & $<0.01$ & N/A & \\
\hline & $(100.00)$ & $(0.00)$ & & & \\
\hline \multirow[t]{2}{*}{ Primary education } & 2,962 & 40 & & 0.21 & $<0.01$ \\
\hline & $(98.3)$ & $(1.67)$ & & $(0.13-0.35)$ & \\
\hline \multirow[t]{2}{*}{ Secondary education } & 8,518 & 221 & & 0.37 & $<0.01$ \\
\hline & $(97.17)$ & $(2.83)$ & & $(0.28-0.48)$ & \\
\hline \multirow[t]{2}{*}{ Higher } & 5,662 & 333 & & 1 & \\
\hline & $(92.63)$ & $(7.37)$ & & & \\
\hline \multirow[t]{2}{*}{ Missing } & 1 & 0 & & & \\
\hline & $(100.00)$ & $(0.00)$ & & & \\
\hline \multicolumn{6}{|c|}{ Educational attainment of partner } \\
\hline \multirow[t]{2}{*}{ No education } & 227 & 2 & $<0.01$ & 1 & \\
\hline & $(99.64)$ & $(0.36)$ & & & \\
\hline \multirow[t]{2}{*}{ Primary education } & 3,556 & 54 & & 4.97 & 0.08 \\
\hline & $(98.22)$ & $(1.78)$ & & $\begin{array}{l}(0.82- \\
30.08)\end{array}$ & \\
\hline
\end{tabular}




\begin{tabular}{|c|c|c|c|c|c|}
\hline & $\begin{array}{l}\text { Never been tested for } \\
\text { HIV }\end{array}$ & $\begin{array}{l}\text { Have been tested for } \\
\text { HIV }\end{array}$ & $\begin{array}{l}\mathrm{p}- \\
\text { value }\end{array}$ & $\begin{array}{l}\text { Crude OR } \\
\text { (with 95\% } \\
\text { Cl) }\end{array}$ & $\begin{array}{l}\text { p-value of } \\
\text { OR }\end{array}$ \\
\hline \multirow[t]{2}{*}{ Secondary education } & 4,999 & 184 & & 13.66 & $<0.01$ \\
\hline & $(95.27)$ & $(4.73)$ & & $\begin{array}{l}(2.28- \\
81.90)\end{array}$ & \\
\hline \multirow[t]{2}{*}{ Higher } & 3,296 & 195 & & 24.14 & $<0.01$ \\
\hline & $(91.93)$ & $(8.07)$ & & $\begin{array}{l}(4.00 \\
-145.58)\end{array}$ & \\
\hline \multirow[t]{2}{*}{ Missing } & 5,296 & 159 & & & \\
\hline & $(96.75)$ & $(3.25)$ & & & \\
\hline \multicolumn{6}{|l|}{ Domicile } \\
\hline \multirow[t]{2}{*}{ Urban } & 5,853 & 378 & $<0.01$ & 1 & \\
\hline & $(93.55)$ & $(6.45)$ & & & \\
\hline \multirow[t]{2}{*}{ Rural } & 11,521 & 216 & & 0.33 & $<0.01$ \\
\hline & $(97.76)$ & $(2.24)$ & & $(0.22-0.49)$ & \\
\hline \multicolumn{6}{|l|}{ Religion } \\
\hline \multirow[t]{2}{*}{ Roman Catholic } & 12,544 & 480 & 0.05 & 1 & \\
\hline & $(95.42)$ & $(4.58)$ & & & \\
\hline \multirow[t]{2}{*}{ Protestant } & 1,659 & 55 & & 0.99 & 0.96 \\
\hline & $(95.48)$ & $(4.52)$ & & $(0.61-1.60)$ & \\
\hline \multirow[t]{2}{*}{ Iglesia ni Cristo } & 507 & 13 & & 0.85 & 0.72 \\
\hline & $(96.09)$ & (3.91) & & $(0.35-2.08)$ & \\
\hline \multirow[t]{2}{*}{ Aglipay } & 244 & 6 & & 0.35 & 0.04 \\
\hline & $(98.35)$ & $(1.65)$ & & $(0.13-0.95)$ & \\
\hline \multirow[t]{2}{*}{ Islam } & 1,560 & 16 & & 0.36 & $<0.01$ \\
\hline & $(98.31)$ & $(1.69)$ & & $(0.19-0.67)$ & \\
\hline \multirow[t]{2}{*}{ Other Christian } & 538 & 11 & & 0.30 & $<0.01$ \\
\hline & $(98.60)$ & $(1.40)$ & & $(0.13-0.70)$ & \\
\hline \multirow[t]{2}{*}{ Other/None } & 322 & 13 & & 0.89 & 0.77 \\
\hline & $(95.91)$ & $(4.09)$ & & $(0.41-1.93)$ & \\
\hline \multicolumn{6}{|c|}{ Access to newspaper or magazine } \\
\hline \multirow[t]{2}{*}{ Not at all } & 8,975 & 200 & $<0.01$ & 1 & \\
\hline & $(97.08)$ & $(2.92)$ & & & \\
\hline \multirow[t]{2}{*}{ Less than once a week } & 6,267 & 242 & & 1.51 & 0.04 \\
\hline & $(95.65)$ & $(4.35)$ & & $(1.02-2.25)$ & \\
\hline \multirow[t]{2}{*}{ At least once a week } & 2,132 & 152 & & 3.05 & $<0.01$ \\
\hline & $(91.60)$ & $(8.40)$ & & $(2.03-4.57)$ & \\
\hline \multicolumn{6}{|l|}{ Access to radio } \\
\hline \multirow[t]{2}{*}{ Not at all } & 5,228 & 130 & $<0.01$ & 1 & \\
\hline & $(97.18)$ & $(2.82)$ & & & \\
\hline \multirow[t]{2}{*}{ Less than once a week } & 6,223 & 218 & & 1.58 & $<0.01$ \\
\hline & $(95.60)$ & $(4.40)$ & & $(1.13-2.22)$ & \\
\hline
\end{tabular}




\begin{tabular}{|c|c|c|c|c|c|}
\hline & $\begin{array}{l}\text { Never been tested for } \\
\text { HIV }\end{array}$ & $\begin{array}{l}\text { Have been tested for } \\
\text { HIV }\end{array}$ & $\begin{array}{l}\mathrm{p}- \\
\text { value }\end{array}$ & $\begin{array}{l}\text { Crude OR } \\
\text { (with 95\% } \\
\text { Cl) }\end{array}$ & $\begin{array}{l}\text { p-value of } \\
\text { OR }\end{array}$ \\
\hline \multirow[t]{2}{*}{ At least once a week } & 5,923 & 246 & & 1.92 & $<0.01$ \\
\hline & $(94.71)$ & $(5.29)$ & & $(1.34-2.77)$ & \\
\hline \multicolumn{6}{|l|}{ Access to television } \\
\hline \multirow[t]{2}{*}{ Not at all } & 1,643 & 15 & $<0.01$ & 1 & \\
\hline & (99.05) & $(0.95)$ & & & \\
\hline \multirow[t]{2}{*}{ Less than once a week } & 3,283 & 106 & & 5.82 & $<0.01$ \\
\hline & $(94.72)$ & $(5.28)$ & & $\begin{array}{l}(2.79- \\
12.11)\end{array}$ & \\
\hline \multirow[t]{2}{*}{ At least once a week } & 12,448 & 473 & & 4.74 & $<0.01$ \\
\hline & $(95.66)$ & $(4.34)$ & & $(2.31-9.71)$ & \\
\hline \multicolumn{6}{|l|}{ Access to internet } \\
\hline \multirow[t]{2}{*}{ Not at all } & 7,764 & 96 & $<0.01$ & 1 & \\
\hline & $(98.39)$ & $(1.61)$ & & & \\
\hline \multirow[t]{2}{*}{ Less than once a week } & 1,823 & 65 & & 2.60 & $<0.01$ \\
\hline & $(95.91)$ & $(4.09)$ & & $(1.72-3.93)$ & \\
\hline \multirow[t]{2}{*}{ At least once a week } & 3,423 & 125 & & 2.11 & $<0.01$ \\
\hline & $(96.66)$ & (3.34) & & $(1.36-3.27)$ & \\
\hline \multirow[t]{2}{*}{ Almost every day } & 4,364 & 308 & & 4.91 & $<0.01$ \\
\hline & $(92.55$ & $(7.45)$ & & $(3.33-7.24)$ & \\
\hline \multicolumn{6}{|l|}{ Wealth index } \\
\hline \multirow[t]{2}{*}{ Poorest } & 4,624 & 33 & $<0.01$ & 1 & \\
\hline & (99.16) & $(0.84)$ & & & \\
\hline \multirow[t]{2}{*}{ Poorer } & 3,936 & 92 & & 2.79 & $<0.01$ \\
\hline & $(97.68)$ & $(2.32)$ & & $(1.54-5.05)$ & \\
\hline \multirow[t]{2}{*}{ Middle } & 3,334 & 134 & & 5.32 & $<0.01$ \\
\hline & $(95.66)$ & $(4.34)$ & & $(2.95-9.59)$ & \\
\hline \multirow[t]{2}{*}{ Richer } & 2,970 & 160 & & 6.19 & $<0.01$ \\
\hline & $(94.99)$ & $(5.01)$ & & $\begin{array}{l}(3.54- \\
10.84)\end{array}$ & \\
\hline \multirow[t]{2}{*}{ Richest } & 2,510 & 175 & & 9.87 & $<0.01$ \\
\hline & $(92.25)$ & $(7.75)$ & & $\begin{array}{l}(5.51- \\
17.66)\end{array}$ & \\
\hline \multicolumn{6}{|c|}{ Work (Employment status) } \\
\hline \multirow[t]{2}{*}{ No } & 9,255 & 252 & $<0.01$ & 1 & \\
\hline & $(97.06)$ & $(2.94)$ & & & \\
\hline \multirow[t]{2}{*}{ Yes } & 8,119 & 342 & & 2.07 & $<0.01$ \\
\hline & $(94.10)$ & $(5.90)$ & & $(1.58-2.72)$ & \\
\hline \multicolumn{6}{|l|}{ Marital Status } \\
\hline \multirow[t]{2}{*}{ Never in union } & $4,643(97.27)$ & 110 & $<0.01$ & 1 & \\
\hline & & $(2.73)$ & & & \\
\hline
\end{tabular}




\begin{tabular}{|c|c|c|c|c|c|}
\hline & $\begin{array}{l}\text { Never been tested for } \\
\text { HIV }\end{array}$ & $\begin{array}{l}\text { Have been tested for } \\
\text { HIV }\end{array}$ & $\begin{array}{l}\text { p- } \\
\text { value }\end{array}$ & $\begin{array}{l}\text { Crude OR } \\
\text { (with 95\% } \\
\text { Cl) }\end{array}$ & $\begin{array}{l}\text { p-value of } \\
\text { OR }\end{array}$ \\
\hline \multirow[t]{2}{*}{ Married } & $9,017(96.35)$ & 256 & & 1.35 & 0.09 \\
\hline & & $(3.65)$ & & $(0.95-1.90)$ & \\
\hline \multirow[t]{2}{*}{ Living with partner } & $3,067(91.67)$ & 180 & & 3.23 & $<0.01$ \\
\hline & & (8.33) & & $(1.93-5.42)$ & \\
\hline \multirow[t]{2}{*}{ Widowed/divorced/separated } & 647 & 48 & & 2.85 & $<0.01$ \\
\hline & $(92.59)$ & $(7.41)$ & & $(1.44-5.64)$ & \\
\hline \multicolumn{6}{|l|}{ Consistent condom use } \\
\hline \multirow{2}{*}{$\begin{array}{l}\text { Did not use condoms during most recent sexual } \\
\text { encounter }\end{array}$} & 11,655 & 432 & 0.18 & 1 & \\
\hline & $(94.73)$ & $(5.27)$ & & & \\
\hline \multirow[t]{2}{*}{ Inconsistent condom use } & 51 & 5 & & 0.95 & 0.93 \\
\hline & $(94.95)$ & $(5.05)$ & & $(0.32-2.84)$ & \\
\hline \multirow[t]{2}{*}{ Consistent condom use } & 190 & 11 & & 2.06 & 0.17 \\
\hline & $(89.72)$ & $(10.28)$ & & $(0.74-5.76)$ & \\
\hline \multirow[t]{2}{*}{ Missing } & 5,478 & 146 & & & \\
\hline & $(97.27)$ & $(2.73)$ & & & \\
\hline \multicolumn{6}{|l|}{ Tobacco use } \\
\hline \multirow[t]{2}{*}{ No } & 16,567 & 547 & 0.03 & 1 & \\
\hline & $(95.87)$ & $(4.13)$ & & & \\
\hline \multirow[t]{2}{*}{ Yes } & 807 & 47 & & 1.86 & 0.03 \\
\hline & $(92.56)$ & $(7.44)$ & & $(1.05-3.32)$ & \\
\hline \multicolumn{6}{|l|}{ Alcohol consumption } \\
\hline \multirow[t]{2}{*}{ No } & 13,145 & 376 & $<0.01$ & 1 & \\
\hline & $(96.76)$ & $(3.24)$ & & & \\
\hline \multirow[t]{2}{*}{ Yes } & 4,229 & 218 & & 2.37 & $<0.01$ \\
\hline & $(92.65)$ & $(7.35)$ & & $(1.77-3.17)$ & \\
\hline
\end{tabular}


Table 2

Continuous Variables and their association with HIV testing.

\begin{tabular}{|c|c|c|c|c|c|c|c|}
\hline & Range & Mean & Median & Distribution & p-value & $\begin{array}{l}\text { Crude OR } \\
\text { (with 95\% Cl) }\end{array}$ & p-value of OR \\
\hline Age of respondent $(n=17,968)$ & $15-49$ & 30.10 & 31 & Right-skewed & $<0.01$ & $\begin{array}{l}1.02 \\
(1.01-1.03)\end{array}$ & $<0.01$ \\
\hline Household size $(n=17,968)$ & $1-21$ & 5.35 & 5 & Right-skewed & $<0.01$ & $\begin{array}{l}0.94 \\
(0.87-1.01)\end{array}$ & 0.10 \\
\hline Age at first sex $(n=13,737)$ & $8-45$ & 20.38 & 19 & Right-skewed & 0.20 & $\begin{array}{l}1.00 \\
(0.97-1.04)\end{array}$ & 0.77 \\
\hline Age of partner $(n=12,334)$ & $10-95$ & 37.14 & 36 & Right-skewed & $<0.01$ & $\begin{array}{l}0.97 \\
(0.96-0.99)\end{array}$ & $<0.01$ \\
\hline Number of lifetime sexual partners $(n=13,998)$ & $1-95+$ & 1.41 & 1 & Right-skewed & $<0.01$ & $\begin{array}{l}1.01 \\
(1.00-1.02)\end{array}$ & 0.17 \\
\hline Number of children $(n=17,968)$ & $0-18$ & 1.77 & 2 & Right-skewed & 0.86 & $\begin{array}{l}0.99 \\
(0.94-1.05)\end{array}$ & 0.75 \\
\hline HIV knowledge $(n=16,382)$ & $0-8$ & 5.18 & 5 & Left-skewed & $<0.01$ & $\begin{array}{l}1.40 \\
(1.25-1.58)\end{array}$ & $<0.01$ \\
\hline $\begin{array}{l}\text { Tolerance to domestic violence score } \\
(n=17,968)\end{array}$ & $0-5$ & 0.21 & 0 & Right-skewed & 0.02 & $\begin{array}{l}0.75 \\
(0.61-0.92)\end{array}$ & $<0.01$ \\
\hline Women's empowerment score $(n=12,384)$ & $0-13$ & 4.82 & 4 & Left-skewed & 0.23 & $\begin{array}{l}0.96 \\
(0.87-1.05)\end{array}$ & 0.36 \\
\hline Extent of controlling behavior of spouse $(n=13,215)$ & $0-5$ & 0.72 & 0 & Right-skewed & 0.21 & $\begin{array}{l}1.06 \\
(0.92-1.22)\end{array}$ & 0.42 \\
\hline
\end{tabular}

We also found that there is strong evidence of association between emotional violence and the following variables: physical violence, sexual violence, any form of violence, hurting one's partner, alcohol intake of partner, having a father who beats her mother, educational attainment of respondent, educational attainment of partner, religion, access to television, wealth index, marital status, tobacco use, alcohol consumption, age at first sexual intercourse, number of lifetime sexual partners, number of children, HIV knowledge score, tolerance to domestic violence score, women's empowerment score, and extent of controlling behavior of spouse (Supplementary File 1). On the other hand, the following variables are associated with physical violence: emotional violence, sexual violence, any form of violence, hurting one's partner, alcohol intake of partner, having a father who beats her mother, educational attainment of respondent, educational attainment of partner, religion, access to internet, wealth index, employment status, marital status, tobacco use, alcohol consumption, age at first sexual intercourse, number of lifetime sexual partners, number of children, tolerance to domestic violence score, women's empowerment score, and extent of controlling behavior of spouse (Supplementary File 2). We also found the following variables are found to be associated with sexual violence: emotional violence, physical violence, any form of violence, hurting partner, alcohol intake of partner, having a father who beats her mother, educational attainment of respondent, educational attainment of partner, domicile, access to internet, wealth index, marital status, employment status, tobacco use, alcohol consumption, age at first sexual intercourse, number of lifetime sexual partners, number of children, tolerance to domestic violence score, women's empowerment score, and extent of controlling behavior of spouse (Supplementary File 3). Lastly, we the following variables are associated with any form of violence: hurting partner, alcohol intake of partner, respondent's father beats her mother, educational attainment of respondent, domicile, religion, wealth index, marital status, employment status, tobacco use, alcohol consumption, age, age at first sexual intercourse, number of lifetime sexual partners, number of children, HIV knowledge score, tolerance to domestic violence score, women's empowerment score, and extent of controlling behavior of spouse (Supplementary File 4). From these cross-tabulations, we control for the effect of the following confounders in the regression model: alcohol intake of partner, educational attainment of respondent, educational attainment of partner, domicile, religion, newspaper, weekly access to radio, tv and internet, wealth index, employment status, marital status, tobacco use, alcohol consumption, age of respondent, household size, age of partner, number of lifetime sexual partners, and tolerance to domestic violence score. Even if women's empowerment score, having a father who beats her mother, and controlling behavior of partner is not associated with our outcome from the data, we will still be controlling for these variables because previous studies have shown that these are known to confound the main association of interest (13-25). We also assessed the potential confounding effect of the other remaining variables through regression modelling, but we did not find any, hence they were not controlled for in our final models. 
Prior to multivariate analysis, we run a correlation matrix to assess potential autocorrelation. We find that the three specific types of IPV variables are correlated with the any type of IPV variable, further providing justification for two separate models. We also excluded respondents who have missing data from the remaining variables of interest, which meant that the multivariate analysis has an effective sample size of 10,265 (50.0\%). After adjusting for confounding, experiencing any form of violence, but not confiding it with anyone lowers the odds of HIV testing by $12 \%$ (aOR: 0.88 ; $95 \%$ Cl: $0.60-1.29$, Table 3) as compared to those who never experienced any form of violence. On the other hand, experiencing any form of violence and confiding it with anyone lowers the odds of HIV testing by $42 \%$ (aOR: 0.58; 95\% Cl: 0.16-2.05). In our model for the specific types of violence, with respect to not experiencing emotional violence, those who have experienced it but have not confided it with anyone increases the odds of HIV testing by 19\% (aOR: 1.19; 95\% Cl: 0.80-1.79, Table 4), while those who experienced emotional violence but have confided it with other people increases the odds of HIV testing by $56 \%$ (aOR: $1.56 ; 95 \% \mathrm{Cl}: 0.44-5.51$ ). We also find that with respect to those who have not experienced physical violence, those who have experienced physical violence but have not confided it with anybody else decreases the odds of HIV testing by $28 \%$ (aOR: $0.72 ; 95 \%$ Cl: $0.35-1.48$ ) while those who confided it with somebody else decreases the odds of HIV testing by $57 \%$ (aOR: $0.43 ; 95 \%$ Cl: 0.07-2.50). Lastly, we also find that with respect to those who have not experienced any form of sexual violence, those who experienced any form of sexual violence but have not confided it with anybody have a $42 \%$ (aOR: $0.58 ; 95 \% \mathrm{Cl}$ : $0.21-1.60$ ) lower odds of HIV testing, while those who have confided it with anybody has a $53 \%$ (aOR: 0.47; $95 \% \mathrm{Cl}$ : $0.06-3.63)$ lower odds of HIV testing. None of these exposure variables showed a statistically significant association with HIV testing, however, urban domicile, weekly access to radio, almost daily access to internet, higher wealth index, living with partner, and high HIV knowledge score are strongly associated with greater odds of HIV testing. 
Table 3

Association of experiencing any form of violence with HIV testing after adjusting for confounding $(\mathrm{n}=$ 10,265).

\begin{tabular}{|c|c|c|c|}
\hline & Adjusted Odds Ratio & 95\% Confidence Interval & p-value \\
\hline \multicolumn{4}{|l|}{ Any Violence } \\
\hline Has not experienced said violence & 1 & & \\
\hline Experienced violence but did not confide & 0.88 & $0.60-1.29$ & 0.52 \\
\hline Experienced violence but confided & 0.58 & $0.16-2.05$ & 0.40 \\
\hline \multicolumn{4}{|l|}{ Alcohol intake of partner } \\
\hline No & 1 & & \\
\hline Yes & 1.31 & $0.88-1.93$ & 0.18 \\
\hline \multicolumn{4}{|l|}{ Educational attainment of respondent } \\
\hline Primary education & 1 & & \\
\hline Secondary education & 1.04 & $0.52-2.05$ & 0.92 \\
\hline Higher & 1.45 & $0.67-3.14$ & 0.34 \\
\hline \multicolumn{4}{|l|}{ Educational attainment of partner } \\
\hline No education & 1 & & \\
\hline Primary education & 1.54 & $0.22-10.74$ & 0.66 \\
\hline Secondary education & 1.62 & $0.24-10.96$ & 0.62 \\
\hline Higher & 1.60 & $0.23-11.02$ & 0.63 \\
\hline \multicolumn{4}{|l|}{ Domicile } \\
\hline Urban & 1 & & \\
\hline Rural & 0.52 & $0.33-0.80$ & $<0.01$ \\
\hline \multicolumn{4}{|l|}{ Access to newspaper or magazine } \\
\hline Not at all & 1 & & \\
\hline Less than once a week & 0.82 & $0.53-1.27$ & 0.38 \\
\hline At least once a week & 1.30 & $0.78-2.17$ & 0.32 \\
\hline \multicolumn{4}{|l|}{ Access to radio } \\
\hline Not at all & 1 & & \\
\hline Less than once a week & 1.49 & $0.91-2.45$ & 0.11 \\
\hline At least once a week & 1.79 & $1.11-2.89$ & 0.02 \\
\hline \multicolumn{4}{|l|}{ Access to television } \\
\hline Not at all & 1 & & \\
\hline Less than once a week & 2.34 & $0.92-5.97$ & 0.07 \\
\hline At least once a week & 2.15 & $0.89-5.19$ & 0.09 \\
\hline \multicolumn{4}{|l|}{ Access to internet } \\
\hline Not at all & 1 & & \\
\hline Less than once a week & 1.44 & $0.83-2.49$ & 0.19 \\
\hline At least once a week & 1.16 & $0.59-2.27$ & 0.67 \\
\hline Almost every day & 2.41 & $1.30-4.49$ & 0.01 \\
\hline \multicolumn{4}{|l|}{ Wealth index } \\
\hline Poorest & 1 & & \\
\hline Poorer & 2.48 & $1.16-5.30$ & 0.02 \\
\hline
\end{tabular}

Page $12 / 19$ 


\begin{tabular}{|c|c|c|c|}
\hline & Adjusted Odds Ratio & 95\% Confidence Interval & p-value \\
\hline Middle & 2.93 & $1.35-6.38$ & 0.01 \\
\hline Richer & 3.27 & $1.49-7.19$ & $<0.01$ \\
\hline Richest & 3.57 & $1.47-8.68$ & 0.01 \\
\hline \multicolumn{4}{|l|}{ Work (Employment status) } \\
\hline No & 1 & & \\
\hline Yes & 1.09 & $0.78-1.53$ & 0.62 \\
\hline \multicolumn{4}{|l|}{ Marital Status } \\
\hline Married & 1 & & \\
\hline Living with partner & 1.91 & $1.23-2.96$ & $<0.01$ \\
\hline \multicolumn{4}{|l|}{ Alcohol consumption } \\
\hline No & 1 & & \\
\hline Yes & 1.16 & $0.80-1.70$ & $0 . .43$ \\
\hline Age of respondent & 0.98 & $0.95-1.01$ & 0.15 \\
\hline Household size & 1.01 & $0.91-1.11$ & 0.91 \\
\hline Age of partner & 1.00 & $0.98-1.02$ & 0.88 \\
\hline Number of lifetime sexual partners & 1.01 & $0.99-1.02$ & 0.48 \\
\hline HIV knowledge & 1.26 & $1.08-1.46$ & $<0.01$ \\
\hline Tolerance to domestic violence score & 0.88 & $0.65-1.18$ & 0.39 \\
\hline \multicolumn{4}{|l|}{ Tobacco use } \\
\hline No & 1 & & \\
\hline Yes & 1.33 & $0.74-2.39$ & 0.35 \\
\hline \multicolumn{4}{|l|}{ Religion } \\
\hline Roman Catholic & 1 & & \\
\hline Protestant & 1.23 & $0.67-2.27$ & 0.50 \\
\hline Iglesia ni Cristo & 0.72 & $0.21-2.47$ & 0.60 \\
\hline Aglipay & 0.26 & $0.06-1.10$ & 0.07 \\
\hline Islam & 1.46 & $0.60-3.51$ & 0.40 \\
\hline Other Christian & 0.43 & $0.12-1.47$ & 0.18 \\
\hline Other/None & 1.02 & $0.42-2.49$ & 0.96 \\
\hline Women's empowerment score & 0.97 & $0.87-1.09$ & 0.64 \\
\hline \multicolumn{4}{|l|}{ Respondent's father beats mother } \\
\hline No & 1 & & \\
\hline Yes & 1.04 & $0.64-1.69$ & 0.88 \\
\hline
\end{tabular}


Table 4

Association of experiencing specific forms of violence with HIV testing after adjusting for confounding ( $\mathrm{n}$ $=10,265$ ).

\begin{tabular}{|c|c|c|c|}
\hline & Adjusted Odds Ratio & 95\% Confidence Interval & p-value \\
\hline \multicolumn{4}{|l|}{ Emotional Violence } \\
\hline Has not experienced said violence & 1 & & \\
\hline Experienced violence but did not confide & 1.19 & $0.80-1.79$ & 0.39 \\
\hline Experienced violence but confided & 1.56 & $0.44-5.51$ & 0.49 \\
\hline \multicolumn{4}{|l|}{ Physical Violence } \\
\hline Has not experienced said violence & 1 & & \\
\hline Experienced violence but did not confide & 0.72 & $0.35-1.48$ & 0.37 \\
\hline Experienced violence but confided & 0.43 & $0.07-2.50$ & 0.34 \\
\hline \multicolumn{4}{|l|}{ Sexual Violence } \\
\hline Has not experienced said violence & 1 & & \\
\hline Experienced violence but did not confide & 0.58 & $0.21-1.60$ & 0.29 \\
\hline Experienced violence but confided & 0.47 & $0.06-3.63$ & 0.47 \\
\hline \multicolumn{4}{|l|}{ Alcohol intake of partner } \\
\hline No & 1 & & \\
\hline Yes & 1.30 & $0.88-1.93$ & 0.19 \\
\hline \multicolumn{4}{|l|}{ Educational attainment of respondent } \\
\hline Primary education & 1 & & \\
\hline Secondary education & 1.02 & $0.51-2.03$ & 0.95 \\
\hline Higher & 1.45 & $0.67-3.15$ & 0.35 \\
\hline \multicolumn{4}{|l|}{ Educational attainment of partner } \\
\hline No education & 1 & & \\
\hline Primary education & 1.63 & $0.23-11.35$ & 0.62 \\
\hline Secondary education & 1.70 & $0.25-11.53$ & 0.58 \\
\hline Higher & 1.69 & $0.25-11.61$ & 0.60 \\
\hline \multicolumn{4}{|l|}{ Domicile } \\
\hline Urban & 1 & & \\
\hline Rural & 0.51 & $0.33-0.79$ & $<0.01$ \\
\hline \multicolumn{4}{|l|}{ Access to newspaper or magazine } \\
\hline Not at all & 1 & & \\
\hline Less than once a week & 0.82 & $0.53-1.28$ & 0.39 \\
\hline At least once a week & 1.29 & $0.77-2.15$ & 0.33 \\
\hline \multicolumn{4}{|l|}{ Access to radio } \\
\hline Not at all & 1 & & \\
\hline Less than once a week & 1.51 & $0.91-2.50$ & 0.11 \\
\hline At least once a week & 1.81 & $1.10-2.97$ & 0.02 \\
\hline \multicolumn{4}{|l|}{ Access to television } \\
\hline Not at all & 1 & & \\
\hline Less than once a week & 2.27 & $0.88-5.81$ & 0.09 \\
\hline At least once a week & 2.10 & $0.87-5.07$ & 0.10 \\
\hline
\end{tabular}




\begin{tabular}{|c|c|c|c|}
\hline & Adjusted Odds Ratio & 95\% Confidence Interval & p-value \\
\hline \multicolumn{4}{|l|}{ Access to internet } \\
\hline Not at all & 1 & & \\
\hline Less than once a week & 1.42 & $0.82-2.48$ & 0.21 \\
\hline At least once a week & 1.14 & $0.57-2.25$ & 0.71 \\
\hline Almost every day & 2.35 & $1.26-4.38$ & $<0.01$ \\
\hline \multicolumn{4}{|l|}{ Wealth index } \\
\hline Poorest & 1 & & \\
\hline Poorer & 2.43 & $1.13-5.22$ & 0.02 \\
\hline Middle & 2.95 & $1.35-6.43$ & 0.01 \\
\hline Richer & 3.38 & $1.53-7.45$ & $<0.01$ \\
\hline Richest & 3.64 & $1.49-8.88$ & 0.01 \\
\hline \multicolumn{4}{|l|}{ Work (Employment status) } \\
\hline No & 1 & & \\
\hline Yes & 1.09 & $0.77-1.53$ & 0.62 \\
\hline \multicolumn{4}{|l|}{ Marital Status } \\
\hline Married & 1 & & \\
\hline Living with partner & 1.90 & $1.23-2.94$ & $<0.01$ \\
\hline \multicolumn{4}{|l|}{ Alcohol consumption } \\
\hline No & 1 & & \\
\hline Yes & 1.15 & $0.79-1.68$ & 0.47 \\
\hline Age of respondent & 0.98 & $0.95-1.01$ & 0.13 \\
\hline Household size & 1.00 & $0.91-1.10$ & 0.94 \\
\hline Age of partner & 1.00 & $0.98-1.02$ & 0.92 \\
\hline Number of lifetime sexual partners & 1.00 & $0.99-1.02$ & 0.65 \\
\hline HIV knowledge & 1.26 & $1.08-1.47$ & $<0.01$ \\
\hline Tolerance to domestic violence score & 0.87 & $0.64-1.17$ & 0.35 \\
\hline \multicolumn{4}{|l|}{ Tobacco use } \\
\hline No & 1 & & \\
\hline Yes & 1.36 & $0.75-2.45$ & 0.31 \\
\hline \multicolumn{4}{|l|}{ Religion } \\
\hline Roman Catholic & 1 & & \\
\hline Protestant & 1.21 & $0.65-2.24$ & 0.55 \\
\hline Iglesia ni Cristo & 0.77 & $0.23-2.63$ & 0.68 \\
\hline Aglipay & 0.27 & $0.06-1.13$ & 0.07 \\
\hline Islam & 1.46 & $0.60-3.52$ & 0.40 \\
\hline Other Christian & 0.41 & $0.12-1.39$ & 0.15 \\
\hline Other/None & 1.02 & $0.42-2.46$ & 0.97 \\
\hline Women's empowerment score & 0.97 & $0.87-1.09$ & 0.59 \\
\hline \multicolumn{4}{|l|}{ Respondent's father beats mother } \\
\hline No & 1 & & \\
\hline
\end{tabular}




\begin{tabular}{|llll|}
\hline & Adjusted Odds Ratio & 95\% Confidence Interval & p-value \\
\hline Yes & 1.04 & $0.64-1.69$ & 0.87 \\
\hline
\end{tabular}

\section{Discussion}

Our analyses showed that while there is a negative association between experiencing any form of violence, as well as physical and sexual violence, with HIV testing, there is a positive association between experiencing emotional violence and HIV testing. Furthermore, there is a lower odds of HIV testing among those who have experienced physical and sexual violence, or any form of IPV and have confided it with anybody as compared to those who have never experienced these specific forms of IPV or any form of IPV.

The factors that were eventually found to be associated with HIV testing (e.g., education, socio-economic status, etc.) were similar with the results to a previous study (21). Furthermore, the lack of association between being a victim of IPV and HIV testing has been previously documented in literature. One previous study explains that this lack of association is the stigma associated with HIV testing, which is further compounded by the stigma associated with IPV. These stigmas, perceived or real, work to prevent availing IPV victims from availing HIV test services (28, 29).

We expect that people who have confided their IPV experiences to anybody would have a higher odds of HIV testing as compared to those who have never experienced IPV or those who have experienced IPV but has never confided it to anybody, however, the results showed that this is only true for people who have experienced emotional violence. More worryingly, those who have experienced physical or sexual violence and have confided these experiences with anybody have a lower odds of HIV testing as compared to those who have never experienced these specific forms of IPV, or even those that have experienced these forms of IPV but have not confided it with anybody. These findings imply that in addition to fear of reprisals, the support systems of IPV victims might not be encouraging enough for them to get HIV testing. Support systems might also be unaware of the need for HIV testing among victims of IPV. Previous studies have highlighted that physical violence and sexual violence have been found to increase the risk of incidence of sexually transmitted infections (30), including HIV in the Philippines (11), highlighting the need to promote access to HIV testing services among victims of IPV (29).

In addition to having a support structure which do not encourage HIV testing, current policies to address violence against women here in the Philippines are also not actively geared towards providing HIV testing services to victims of IPV. A review of the Anti-Violence Against Women and Their Children Act of 2004, and the handbook for its grassroots-level implementation, the Barangay Violence Against Women Desk Handbook (English), reveals that only referrals to other facilities are provided according to the needs of the client $(31,32)$. By not having a clear-cut indication for counselling nor for testing HIVs and other sexually-transmitted infections (STIs), this could represent a missed opportunity to tell the respondent about the risks of HIVs and STIs and the need for testing for it, as well as in identifying cases of HIV in a high-risk population $(10,11,30)$. The World Health Organization clinical and policy guidelines on responding to intimate partner violence recommends that there should be HIV testing is recommended before giving HIV post-exposure prophylaxis (HIV-PEP), but a negative result should not preclude a client from receiving HIV-PEP. It further recommends that considering local prevalence, ethical and resource and constraints, HIV-PEP should be routinely offered as part of the standard of care for rape victims (33). In the Philippines where the cases are rapidly increasing, there could be some benefit if victims of IPV would receive counselling on the harms of HIV and other STIs upon consultation with frontline care providers, healthcare professionals or police. They should also be routinely offered testing for HIV and other STIs, as well as HIV-PEP and PEP for other STIs as part of the standard of care. Lastly, interventions addressing both IPV and HIV should: (1) consider involvement of relevant end-users; (2) strengthen the capacity of women to act independently and make their own choices; (3) and should consider technological developments and changing demographics for it to be successful (34).

\section{Limitations}

Our study has several limitations. The exclusion of some $50 \%$ of the respondents due to missing data could contribute to the lack of statistical significance and possible selection bias due to missing data. The respondents that are usually excluded have never had a partner, and so had missing data for all 'partner' variables. This could also limit the generalizability of the findings of the study to women who have had a partner. There could be unmeasured confounding as some factors, such as fear of positive result, stigma, pregnancy status, and other institutional factors were not considered in this analysis. Lastly, there could be reverse causality for variables which could change with time, such as the main exposure variables and the other score variables controlled for in the analysis.

\section{Conclusions}

Around $16 \%$ of respondents reported experiencing any form of IPV, $13 \%$ have reported experiencing emotional violence, $7 \%$ have reported experiencing physical violence, and $3 \%$ reported experiencing sexual violence, with an even smaller percentage confiding it with other people. Only $4 \%$ of respondents have ever had tested for HIV. Those who have experienced emotional violence have a higher odds of HIV testing as compared to those who have never experienced emotional violence, and even higher when it is confided to another person. However, the odds of HIV testing are lower among those who have experienced any form of violence, physical violence, and sexual violence as compared to respondents who have not experienced any form of violence, or these specific types of violence. More worryingly, the odds of HIV testing among victims of these types of IPV and have confided with other people are lower. Despite having non-significant results, these results underline the need to improve access to HIV testing services among victims of IPV 
by making tests for HIV and other STIs part of the standard of care for IPV victims. This could be done by counselling victims of IPV on the dangers of HIV and STI and offering them routinely testing for HIV and STIs as well as PEPs for HIV and other STIs. Furthermore, there may also be a need to conduct further research to improve support systems of IPVs, their access to professional help through SMS and/or social media, and other interventions to improve uptake of uptake of testing for HIV and other STIs. By doing so, we help reach the 'first 90' of the 90-90-90 UNAIDS targets in a very vulnerable population and enable IPVs to know about their true HIV status.

\author{
Abbreviations \\ HIV - Human Immunodeficiency Virus \\ PEP - Post exposure prophylaxis \\ STI - Sexually Transmitted Infections \\ NDHS - National Demographic and Health Survey \\ UNAIDS - Joint United Nations Program on HIV/AIDS
}

\title{
Declarations
}

\section{Ethics approval and consent to participate}

The study has undergone ethics approval at the Ateneo de Manila University Research Ethics Committee (Protocol ID: SMPH DATA 2020).

\section{Consent for publication}

Not applicable.

\section{Availability of data and materials}

The data for the 2017 Philippine National Demographic and Health Survey Individual Recode are available from the Demographic and Health Surveys Program Website (https://www.dhsprogram.com/data/available-datasets.cfm).

\section{Competing Interests}

The authors declare no competing interests.

\section{Funding}

This study is supported by the Ateneo de Manila University Research Council Standard Grant (Grant No.: 2020-01).

\section{Contributions}

VCFP, AMLA, and LBST conceived the study. VCFP and AMLA performed the statistical analysis. VCFP, CST, JRM, NAA, EJMN, NAKA wrote the first draft. AMLA, SCCG, MMO, SJDLR and LBST gave feedback and reviewed the drafts of the manuscript. LBST is the senior author of the study. All authors read and approved the final manuscript.

\section{Acknowledgments}

We thank the Demographic and Health Survey Program for allowing us to use their datasets to carry out this project.

\section{References}

1. Intimate Partner Violence |Violence Prevention|Injury Center|CDC [Internet]. [cited 2021 Feb 21]. Available from: https://www.cdc.gov/violenceprevention/intimatepartnerviolence/index.html

2. Frank TD, Carter A, Jahagirdar D, Biehl MH, Douwes-Schultz D, Larson SL, et al. Global, regional, and national incidence, prevalence, and mortality of HIV, 1980-2017, and forecasts to 2030, for 195 countries and territories: a systematic analysis for the Global Burden of Diseases, Injuries, and Risk Factors Study 2017. Lancet HIV. 2019 Dec 1;6(12):e831-59.

Page 17/19 
3. Murray CJL, Vos T, Lozano R, Naghavi M, Flaxman AD, Michaud C, et al. Disability-adjusted life years (DALYs) for 291 diseases and injuries in 21 regions, 1990-2010: a systematic analysis for the Global Burden of Disease Study 2010. Lancet. 2012 Dec 15;380(9859):2197-223.

4. Salvana E. The Philippine HIV/AIDS Epidemic: A Call to Arms. Act Med Philipp. 2010;44(1):60-2.

5. Farr AC, Wilson DP. An HIV epidemic is ready to emerge in the Philippines. J Int AIDS Soc. 2010;13(1):16-16.

6. Department of Health (Philippines) Epidemiology Bureau. The State of the Philippine HIV Epidemic 2016: Facing Challenges, Forging Solutions. 2016. Available from:

http://www.doh.gov.ph/sites/default/files/publications/publication_nonSerials_State\%20of\%20HIV\%20Epidemic\%20in\%20the\%20Philippines.pdf

7. Gohil J, Baja ES, Sy TR, Guevara EG, Hemingway C, Medina PMB, et al. Is the Philippines ready for HIV self-testing? BMC Public Health. 2020 Jan 9;20(1):34.

8. Health Technology Assessment Study Group - Health Policy Development and Planning Bureau. Rapid HIV Diagnostic Algorithm (rHIVda) for the Philippines. Department of Health (Philippines); 2018. Available from: https://www.doh.gov.ph/sites/default/files/publications/IB_rHIVda.pdf

9. Philippine Statistics Authority (PSA), ICF. Philippine National Demographic and Health Survey 2017 [Internet]. PSA and ICF; 2018. Available from: https://psa.gov.ph/sites/default/files/PHILIPPINE\%20NATIONAL\%20DEMOGRAPHIC\%20AND\%20HEALTH\%20SURVEY\%202017_new.pdf

10. Li Y, Marshall CM, Rees HC, Nunez A, Ezeanolue EE, Ehiri JE. Intimate partner violence and HIV infection among women: a systematic review and meta-analysis. J Int AIDS Soc. 2014;17(1):18845.

11. Lucea MB, Hindin MJ, Kub J, Campbell JC. HIV Risk, Partner Violence, and Relationship Power Among Filipino Young Women: Testing a Structural Model. Health Care Women Int. 2012 Apr 1;33(4):302-20.

12. Hindin MJ, Adair LS. Who's at risk? Factors associated with intimate partner violence in the Philippines. Soc Sci Med. 2002 Oct;55(8):1385-99.

13. Santambrogio J, Colmegna F, Trotta G, Cavalleri PR, Clerici M. [Intimate partner violence (IPV) and associated factors: an overview of epidemiological and qualitative evidence in literature] - PubMed. Riv Psichiatr. 2019;54(3):97-108.

14. Alebel A, Kibret GD, Wagnew F, Tesema C, Ferede A, Petrucka P, et al. Intimate partner violence and associated factors among pregnant women in Ethiopia: a systematic review and meta-analysis. Reprod Health. 2018;15:196.

15. Bacchus LJ, Ranganathan M, Watts C, Devries K. Recent intimate partner violence against women and health: a systematic review and metaanalysis of cohort studies. BMJ Open. 2018;8(7). Available from: https://bmjopen.bmj.com/content/8/7/e019995

16. Fox A, Jackson SS, Hansen N, Gasa N, Crewe M, Sikkema KJ. In Their Own Voices: A Qualitative Study of Women's Risk for Intimate Partner Violence and HIV in South Africa. Violence Against Women. 2007;13(6):583-602.

17. Kerridge B, Tran P. Husband/Partner Intoxication and Intimate Partner Violence Against Women in the Philippines. Asia Pac J Pub Health. 2016;28(6):507-18.

18. Jiwatram-Negron T, El-Bassel N, Primbetova S, Terlikbayeva A. Gender-Based Violence Among HIV-Positive Women in Kazakhstan: Prevalence, Types, and Associated Risk and Protective Factors. Violence Against Women. 2018;24(3):1570-90.

19. Durevall D, Lindskog A. Intimate partner violence and HIV in ten sub-Saharan African countries: what do the Demographic and Health Surveys tell us? Lancet Glob Health. 2015 Jan;3(1):e34-43.

20. Yabukovich AR, Stockl H, Murray J, Melendez-Torres GJ, Steinert JI, Glavin CEY, et al. Risk and Protective Factors for Intimate Partner Violence Against Women: Systematic Review and Meta-analyses of Prospective-Longitudinal Studies. Am J Pub Health. 2018;108(7):e1-11.

21. Pepito VCF, Newton S. Determinants of HIV testing among Filipino women: Results from the 2013 Philippine National Demographic and Health Survey. PLOS ONE. 2020 May 12;15(5):e0232620.

22. Deblonde J, De Koker P, Hamers FF, Fontaine J, Luchters S, Temmerman M. Barriers to HIV testing in Europe: a systematic review. Eur J Pub Health. 2010;20(4):422-32.

23. Nasrullah M, Oraka E, Breiding MJ, Chavez PR. HIV Testing and Intimate Partner Violence Among Non-Pregnant Women in 15 US States/Territories: Findings from Behavioral Risk Factor Surveillance System Survey Data. AIDS Behav. 2013 Sep 1;17(7):2521-7.

24. Schaffer EM, Agot K, Thirumurthy H. The Association between Intimate Partner Violence and Women's Distribution and Use of HIV Self-Tests with Male Partners: Evidence from a Cohort Study in Kenya. J Acquir Immune Defic Syndr. 2017 Nov 1;76(3):e85-7.

25. Maher JE, Peterson J, Hastings K, Dahlberg LL, Seals B, Shelley G, et al. Partner violence, partner notification, and women's decisions to have an HIV test. J Acquir Immune Defic Syndr. 2000 Nov 1;25(3):276-82.

26. StataCorp. Stata 14.0 IC. College Station, TX: StataCorp; 2015.

27. Rothman KJ. No adjustments are needed for multiple comparisons. Epidemiology. 1990 Jan;1(1):43-6.

28. Turan JM, Bukusi EA, Onono M, Holzemer WL, Miller S, Cohen CR. HIV/AIDS stigma and refusal of HIV testing among pregnant women in rural Kenya: results from the MAMAS Study. AIDS Behav. 2011 Aug;15(6):1111-20.

29. Etudo O, Metheny N, Stephenson R, Kalokhe AS. Intimate partner violence is linked to less HIV testing uptake among high-risk, HIV-negative women in Atlanta. AIDS Care. 2017 Aug;29(8):953-6.

30. Allsworth JE, Anand M, Redding C, Peipert JF. Physical and Sexual Violence and Incident Sexually Transmitted Infections. J Womens Health (Larchmt). 2009;18(4):529-34. 
31. Philippine Commission on Women. Barangay VAW Desk Handbook (English). Philippine Commission on Women; 2015. Available from: https://napc.gov.ph/sites/default/files/gad/Brgy.VAW\%20Handbook_English\%20Final\%20Cover\%20and\%20Texts.pdf

32. Philippine Commission on Women. Republic Act 9262: Anti-Violence Against Women and Their Children Act of 2004. 2004. Available from: https://pcw.gov.ph/republic-act-9262-anti-violence-against-women-and-their-children-act-of-2004/

33. World Health Organization, editor. Responding to intimate partner violence and sexual violence against women: WHO clinical and policy guidelines. Geneva: World Health Organization; 2013. 55 p.

34. Mannell J, Willan S, Shahmanesh M, Seeley J, Sherr L, Gibbs A. Why interventions to prevent intimate partner violence and HIV have failed young women in southern Africa. J Int AIDS Soc. 2019;22(8):e25380.

\section{Supplementary Files}

This is a list of supplementary files associated with this preprint. Click to download.

- IPVandHIVTestingSF1.docx

- IPVandHIVTestingSF2.docx

- IPVandHIVTestingSF3.docx

- IPVandHIVTestingSF4.docx 